\title{
External Feedback Sensitivity of Partly Gain-Coupled DFB Semiconductor Lasers
}

\author{
R. Hui, M. Kavehrad, and T. Makino
}

\begin{abstract}
External optical feedback sensitivity of partly gaincoupled DFB semiconductor lasers has been analyzed in above threshold operation regime. Both the longitudinal spatial hole burning and the nonlinear gain compression have been taken into account. A comparison has been made among $\lambda / 4$-shifted, pure index-coupled and partly gain-coupled DFB laser diodes. Even though pure index-coupled and partly gain coupled DFB lasers exhibit similar sensitivity to external optical feedback at the threshold, however, gain grating can reduce the feedback sensitivity when the lasers operate well above the threshold specially when the $\kappa L$ parameter is high.
\end{abstract}

\section{INTRODUCTION}

$\mathbf{T}$ HE EFFECT OF external optical feedback on the semiconductor lasers has been an important issue for over a decade now [1], [2] and many efforts have been made to reduce the effect of unwanted external optical feedback. Partly gain-coupled DFB lasers have recently received considerable attention because of their remarkable properties [3]-[6]. Apart from the well known high single-longitudinal mode yield, experiments show that the introduction of partly gain coupling mechanism can reduce the external optical feedback sensitivity [7], [8]. This later property is also important in many practical applications specially when a laser diode is monolithically integrated with an external optical modulator or an optical amplifier. External optical feedback on DFB lasers has been studied extensively both in the index-coupled and in the gaincoupled [9]-[11] cases. Theoretical results in [10], [11] indicate, however, that the external feedback sensitivity in partlygain coupled lasers is not significantly reduced in comparison with the pure index-coupled lasers. This is in agreement in general as concerning feedback sensitivity [12]. All these theoretical works so far have been limited by the assumption that lasers operated at threshold and therefore the spatial holeburning effect was be neglected. In this letter, we analyze the effect of weak optical feedback on DFB laser diodes operating above threshold where the longitudinal spatial holeburning and thus the axial carrier density nonuniformity has been taken into account. The results reveal that even though pure index grating and partly gain grating DFB lasers exhibit similar sensitivity to external optical feedback at the threshold, gain grating has the effect of reducing the feedback sensitivity

Manuscript received April 20, 1994; revised May 27, 1994. This work was supported by the Telecommunication Research Institute of Ontario, Photonics Networks and Systems Thrust.

R. Hui and M. Kavehrad are wi th the Electrical Engineering Department, University of Ottawa, Ottawa, ON, K1N 6N5 Canada.

T. Makino is with the Advanced Technology Lab., Bell-Northern Research, Ottawa, ON, K1Y 4H7 Canada.

IEEE Log Number 9403619. when the lasers operate well above threshold especially when the $\kappa L$ parameter is sufficiently high.

\section{ANALYSIS}

The sensitivity to external optical feedback on semiconductor lasers operating at threshold has been analyzed by Favre [9] who introduced feedback sensitivity factor $C$ defined by $C_{R, L}=-2 r_{R, L} \exp (-j \phi) /\left(j \Delta \omega \tau_{\text {int }}\right)$, where the subscripts $R$ and $L$ indicate the reflections are to the right or to the left facet of the laser diode, $\Delta \omega$ is the complex frequency change induced by a small amount of external reflection $r_{R, L}$ with the phase delay $\phi$ and $\tau_{\text {int }}$ is the round trip group delay of the solitary laser diode. At low feedback levels, $C_{R, L}$ depends only on the characteristics of the laser diode itself. By direct computation, it has been demonstrated [8] that $\left|C_{L}\right| / P_{L}=\left|C_{R}\right| / P_{R}$, where $P_{L}$ and $P_{R}$ are the output optical power from the left and the right facets of the laser diode. This relationship can be easily understood because, intuitively, stronger feedback effect will be associated with the laser facet having a higher photon density.

Let $z$-axis be along the laser cavity and the laser is extended from $z=0$ to $z=L$. The electric field can be written as the sum of the forward-going and backward-going waves $E(z)=R(z) \exp \left(-j \beta_{0}(z)\right)+S(z) \exp \left(j \beta_{0}(z)\right)$ with $\beta_{0}=$ $\pi / \Lambda$ and $\Lambda$ denotes the grating period. $R(z)$ and $S(z)$ obey the coupled-wave equation [3]

$$
\begin{aligned}
& d R(z) / d z=[g(z)-j \delta(z)] R(z)-j \kappa_{R S} S(z) \\
& d S(z) / d z=-[g(z)-j \delta(z)] S(z)+j \kappa_{S R} R(z) .
\end{aligned}
$$

In these equations, $g(z)$ is the model gain, $\delta(z)$ is the normalized frequency detuning defined as $\delta(z)=2 \pi n_{\mathrm{eff}}(z) / \lambda-\pi / \Lambda$, $n_{\mathrm{eff}}(z)$ is the effective refractive index and $\lambda$ is the oscillation wavelength. Parameters $\kappa_{R S}$ and $\kappa_{S R}$ represent the grating coupling coefficients. For most partly gain-coupled DFB lasers it has been proven [13] that $\kappa_{R S}=\kappa_{S R}=k \exp (j \psi)$ with the real and the imaginary parts representing the index and the gain grating, respectively.

The steady-state carrier distribution $N(z)$ can be described by the rate equation as:

$$
I / e V-N(z) / \tau_{e}-v_{g} a\left[N(z)-N_{0}\right][1-\varepsilon P(z)] P(z)=0
$$

where $\tau_{e}$ is the effective carrier life time, $V$ is the volume of the active region, $v_{g}$ is the group velocity, $N_{0}$ is the carrier density at transparency, $I$ is the injection current, $e$ is the electron charge, $\varepsilon$ represents the effect of the nonlinear gain compression, $a$ is the differential gain, and $P(z)$ is the 
photon distribution related to $R(z)$ and $S(z)$ as $P(z)=$ $\left(|R(z)|^{2}+|S(z)|^{2}\right) / h \nu$ with $h \nu$ the photon energy. Since the carrier density $N(z)$ is nonuniform along the laser cavity, $g$ and $\delta$ should be $z$-dependent as well, that is

$$
\begin{aligned}
& g(z)=\Gamma(a / 2)\left[N(z)-N_{0}\right][1-\varepsilon P(z)]-\alpha_{0} \\
& \delta(z)=\delta_{0}-\alpha \Gamma(a / 2)\left[N(z)-N_{\mathrm{th}}\right]
\end{aligned}
$$

where $\alpha_{0}$ is the cavity loss coefficient, $N_{\text {th }}$ is the threshold carrier density, $\delta_{0}=2 \pi n_{0} / \lambda-\pi / \Lambda$ is the normalized frequency detuning at the threshold and $\alpha$ is the linewidth enhancement factor of the laser diode.

Suppose there is an external mirror with an amplitude reflectivity $\rho_{\text {ext }}$ facing the right facet of the laser, the boundary condition at the left facet is $R(0)=\rho_{L} \exp \left(-j \phi_{L}\right) S(0)$ while at the right facet, it will be $S(L)=\rho_{\text {eff }} \exp \left(-j \phi_{R}\right) R(L)$ with $\rho_{\text {eff }}=\rho_{R}+\left(1-\rho_{R}^{2}\right) \rho_{\text {ext }} \exp \left[-j\left(\omega \tau+\phi_{\text {ext }}\right)\right] . \rho_{L}, \rho_{R}, \phi_{L}$, and $\phi_{R}$ being the reflectivities and the grating phases at the laser facets, $\tau$ being the round-trip delay of the external cavity and $\phi_{\text {ext }}$ being the phase shift of the external reflector. Equations (1)-(3) can be solved numerically under the given boundary conditions. The calculation procedures are similar to those used in [14], [15]. The transmission matrix method is used and the laser cavity is divided into 200 sections in our calculations. In each section the carrier density $N$ and the photon density $P$ are assumed to be a constant. By an initial guess on the values of $\lambda$ and $S(0)$, the equations can be solved by an iterative method. The complex feedback sensitivity defined in [9] is:

$$
|C|=|\Delta g-j \Delta \delta| L / \rho_{\text {ext }}
$$

where $\Delta g$ and $\Delta \delta$ are the gain and the detuning difference between the solitary laser and the laser with external optical feedback, respectively. In our calculations, however, since the spatial variation of both the gain and the detuning have been taken into account, $\Delta g L$ and $\Delta \delta L$ have to be expressed in their integral form. Therefore, the feedback sensitivity parameter can be obtained from:

$$
\begin{aligned}
|C|= & {\left[\left|\int_{0}^{L} g_{r}(z) d z-\int_{0}^{L} g_{0}(z) d z\right|^{2}+\right.} \\
& \left.\left|\int_{0}^{L} \delta_{r}(z) d z-\int_{0}^{L} \delta_{0}(z) d z\right|^{2}\right]^{1 / 2} / \rho_{\text {ext }}
\end{aligned}
$$

with the subscripts $r$ and 0 representing the quantities with and without the external optical feedback. Since the external feedback sensitivity is usually proportional to the output optical power from the same facet as shown before, the normalized value $|C| P_{\text {out }}$ is also an important parameter to the laser performance. The output optical power $P_{\text {out }}$ can be easily obtained from the calculated value of $|R(L)|^{2}$. Laser parameters used in our calculation are: $a=2.8 \times 10^{-20} \mathrm{~m}^{2}$, $N_{0}=10^{24} \mathrm{~m}^{-3}, \varepsilon=5 \times 10^{-24} \mathrm{~m}^{3}, \alpha_{0}=2.1 \times 10^{3} \mathrm{~m}^{-1}$, $\Lambda=0.24 \mu \mathrm{m}, n_{0}=3.23, L=300 \mu \mathrm{m}, \Gamma=0.25$, and $\tau_{e}=1$ ns.

Fig. 1 shows the calculated feedback sensitivity $|C|$ versus the normalized injection current, for a $\lambda / 4$ shifted DFB laser, a pure index coupled DFB laser and partly gain-coupled DFB lasers with different $\Psi$ values. The plots start from

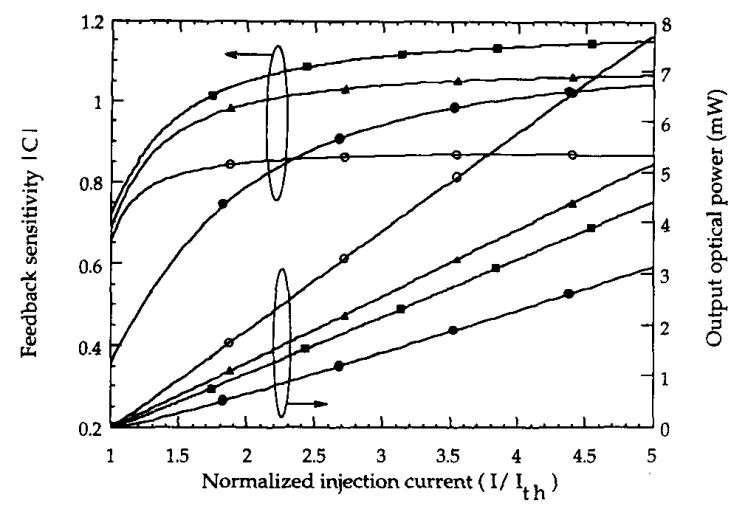

Fig. 1. $|C|$ parameters and output optical power versus the normalized injection current for $\lambda / 4$ shifted (solid points), pure index-coupled (solid rectangles) and partly gain coupled DFB lasers (solid triangles for $\Psi=10^{\circ}$ and open cycles for $\Psi=20^{\circ}$ ). $\alpha=6$ and $\kappa L=3$ are used in this plot.

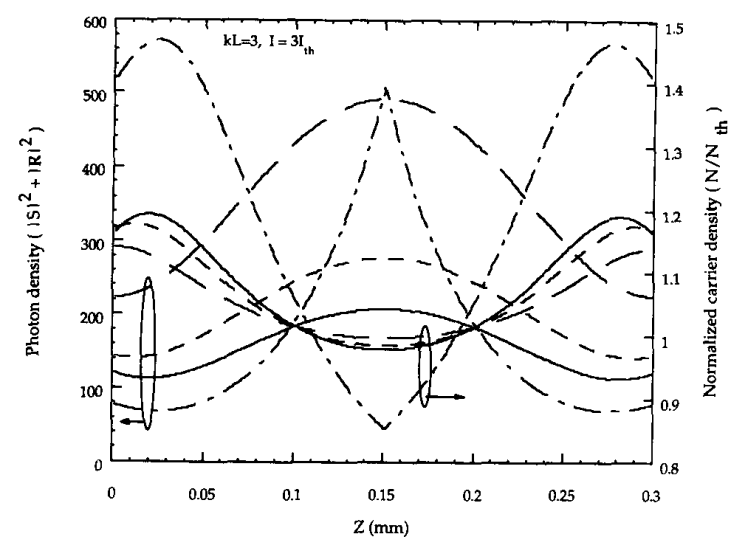

Fig. 2. Photon density and carrier density distributions along the cavity longitudinal direction for $\lambda / 4$ shifted (dot-dashed lines), pure index-coupled (solid lines) and partly gain coupled DFB lasers (short-dashed lines for $\Psi=10^{\circ}$ and long-dashed lines for $\left.\Psi=20^{\circ}\right) \cdot \kappa L=3$ and $I=3 I_{\mathrm{th}}$ are used in this plot.

the threshold points with the values in agreement with the results given by Favre [9], [10]. However, above threshold, the parameter $|C|$ of the $\lambda / 4$ shifted DFB laser exhibits the highest sensitivity to the bias level while partly gain-coupled DFB lasers are relatively insensitive to the bias level specially when the $\Psi$ value is high, that is, with a higher percentage of gain coupling. The physical reason behind the increase in the $|C|$ value above threshold is believed to be the longitudinal spatial hole burning effect. Fig. 2 shows the longitudinal photon density and carrier density distribution along the laser cavity for the four lasers as in Fig. 1 with the bias level fixed at $I=3 I_{\mathrm{th}}$. It is obvious from this figure that the $\lambda / 4$ shifted DFB laser has the most strong spatial hole burning effect and since photons are piled up near the phase shift region, the output optical power is relatively small. It is interesting to note that even though the partly gain-coupled DFB laser have a higher output efficiency, their carrier distribution along the cavity are more uniform and thus less spatial hole burning than their pure index-coupled counterpart. Therefore, the $|C|$ values of partly gain-coupled DFB lasers exhibit less sensitivity to 


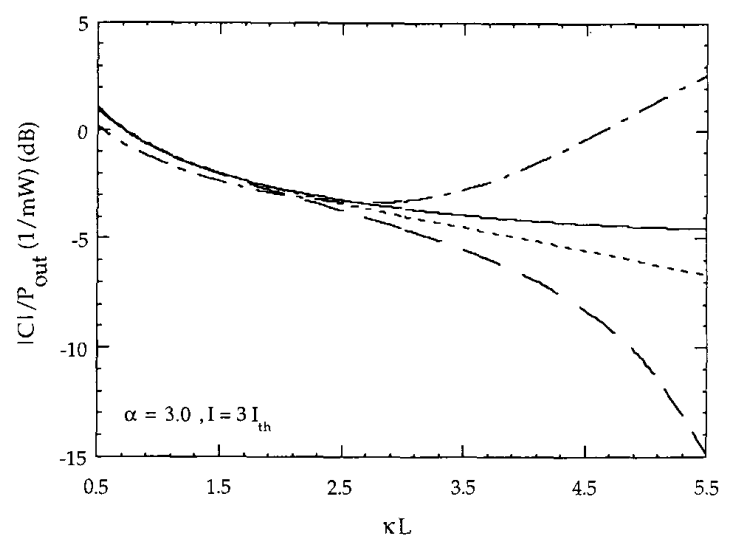

Fig. 3. $|C| / P_{\text {out }}$ versus $\kappa L$ for $\lambda / 4$ shifted (dot-dashed lines), pure index-coupled (solid lines) and partly gain coupled DFB lasers (short-dashed lines for $\Psi=5^{\circ}$ and long-dashed lines for $\Psi=10^{\circ}$ ). $\alpha=3$ and $I=3 I_{\mathrm{th}}$ are used in this plot.

the bias level as shown in Fig. 1. Although the $|C|$ value of the $\lambda / 4$ shifted DFB laser is lower in most part of Fig. 1, its output optical power is also the smallest. Therefore, the trade-off between the $|C|$ value and the output power, i.e., $|C| P_{\text {out }}$, is the most important parameter to use in comparing different laser structure and this parameter can be referred to as the normalized feedback sensitivity. Obviously, partly gaincoupled DFB lasers have lower $|C| P_{\text {out }}$ values compared to both pure index-coupled and $\lambda / 4$ shifted DFB lasers and this can easily be seen from Fig. 1 .

Fig. 3 shows the normalized feedback sensitivity versus the coupling coefficient $\kappa L$ for different laser structures. No apparent difference can be observed with $k L<3$. However, with higher $\kappa L$ values, the normalized feedback sensitivity degrades for the $\lambda / 4$ shifted DFB laser because of the strong spatial hole burning while this performance is improved for partly gain-coupled DFB lasers. Up to $10 \mathrm{~dB}$ improvement has been found for a DFB laser with $\sim 18 \%\left(\Psi=10^{\circ}\right)$ gaincoupling included and with the $\kappa L$ value of 5.5. Therefore, the introduction of partly gain coupling in DFB lasers with a high $\kappa L$ is helpful in reducing the normalized feedback sensitivity.

\section{CONCLUSION}

We have compared the external optical feedback sensitivity of $\lambda / 4$ shifted, pure index-coupled and partly gain-coupled
DFB lasers when they are operating above threshold. Both the longitudinal spatial hole burning and the nonlinear gain comparison have been taken into account. Partly gain coupling in DFB lasers with high $\kappa L$ is less sensitive to external optical feedback.

\section{REFERENCES}

[1] R. Lang and K. Kobayshi, "External optical feedback effects on semiconductor injection laser properties," IEEE J. Quantum Electron., vol. QE-16, pp. 347-355, 1980.

[2] R. W. Tkach and A. R. Chraplyvy, "Regimes of feedback effects in 1.5$\mu \mathrm{m}$ distributed feedback lasers," IEEE J. Lightwave Tech., vol. LT-4, pp. $1655-1661,1986$.

[3] H. Kogelnik and C. V. Shank, "Coupled wave theory of distributed feedback lasers," J. Appl. Phys. vol. 42, pp. 2327-2335, 1972.

[4] Y. Luo, Y. Nakano, K. Ikeda, K. Tada, T. Inoue, H. Hosomastu, and $\mathrm{H}$. Iwaoka, "Low threshold $\mathrm{CW}$ operation in a novel gain-coupled distributed feedback semiconductor laser," in Proc. 12th IEEE Int. Semiconductor Laser Conf., Davos, 1990, pp. 70-71.

[5] B. Borchert, K. David, B. Stegmuller, R. Gessner, M. Beschorner, D. Sacher, and G. Franz, "1.55- $\mu \mathrm{m}$ gain-coupled quantum-well distributed feedbacklasers with high singlemode yield and narrow linewidth," IEEE Photon. Tech. Lett., vol. 3, pp. 955-957, 1992.

[6] G. P. Li, T. Makino, and H. Lu, "Simulation and interpretation of longitudinal-mode behavior in partly gain-coupled InGaAsP/InP multiquantum-well DFB lasers," IEEE Photon. Tech. Lett., vol. 4, pp. 386-389, 1993.

[7] Y. Nakano, Y. Deguchi, K. Ikeda, Y. Luo, and K. Tada, "Resistance to external optical feedback in a gain-coupled semiconductor DFB laser," in Proc. 12th IEEE Int. Semiconductor Laser Conf., Da vos, 1990, pp. $72-73$.

[8] - , "Reduction of excess intensity noise induced by external reflection in a gain-coupled distributed feedback semiconductor laser," IEEE $J$. Quantum Electron., vol. 27, pp. 1732\&nd ash;1735, 1991

[9] F. Favre, "Theoretical analysis of external optical feedback on DFB semiconductor lasers," IEEE J. Quantum Electron., vol. QE-23, pp. $81-88,1987$.

[10] F. Favre, "Sensitivity to external feedback for gain-coupled DFB semiconductor lasers," Electron. Lett., vol. 27, pp. 433-435, 1991.

[11] M. Suhara, S. Islam, and M. Yamada, "Criterion of external feedback sensitivity in index-coupled and gain-coupled DFB semiconductor lasers to be free from excess intensity noise," IEEE J. Quantum Electron., vol. 30, pp. 3-8, 1994.

[12] O. Nilsson and J. Buus, "Linewidth and feedback sensitivity of semiconductor diode lasers," IEEE J. Quantum Electron., vol. 26, pp. 2039-2042, 1990.

[13] K. David, J. Buus, and R. G. Baets, "Basic analysis of AR-coated, partly gain-coupled DFB lasers: The standing wave effect," IEEE J. Quantum Electron., vol. 28, pp. 427-433, 1992.

[14] K. Kikuchi and H. Tomofugi, "Analysis of oscillation characteristics of separated-electrode DFB laser diodes," IEEE J. Quantum Electron., vol. 26, pp. $1717-1727,1990$

[15] H. J. Wu and H. C. Chang, "Analysis of external optical feedback on distributed-feedback semiconductor lasers above threshold," IEEE Photon. Tech. Lett., vol. 5, pp. 1168-1170, 1993. 